\title{
Development of an oral tolerance model in rats for investigation of bioactive food components
}

\author{
M. Jacobsen, H. R. Juul-Madsen and C. Lauridsen \\ Institute of Animal Health and Bioscience, Research group in Immunology and Microbiology, Faculty of Agricultural \\ Science, Aarhus University, 8830 Tjele, Denmark
}

In a recent study on rats, complete diets produced from three cultivation systems were compared on a range of health biomarkers, and notable differences appeared with regard to $\operatorname{IgG}$ levels ${ }^{(1)}$. In order to chase this result, the objective of the present study was to develop an oral tolerance model in rats for comparison of diets composed of ingredients from different organic and conventional cultivation systems with regard to immunological responses.

The oral tolerance model was designed to test if oral tolerance could be developed in suckling rat pups after provision of an antigen to the dam ${ }^{(2)}$. Three treatment groups of lactating rats with two dams in each group were established. In two groups, OVA (ovalbumine, $200 \mathrm{mg} / \mathrm{d} / \mathrm{dam}$ ) was administered to the drinking water during day 10-16 of the lactating period, and the litter size of each dam consisted of either four or eight pups (+OVA/4 pups and +OVA/8 pups, respectively). The third group was given regular tap water, and had four pups per dam (-OVA/4 pups). To test if oral tolerance was induced, the pups were immunized post-weaning with OVA $(80 \mu \mathrm{g} / \mu \mathrm{l}$ complete Freunds adjuvant/isotonic $\mathrm{NaCl}(1: 1) / \mathrm{rat})$ and boosted with OVA $(80 \mu \mathrm{g} / \mu 1$ incomplete Freunds adjuvant/isotonic $\mathrm{NaCl}(1: 1) /$ rat). Blood samples were drawn from week 3 to 11. An ELISA protocol was established for measuring anti-OVA IgG in plasma.

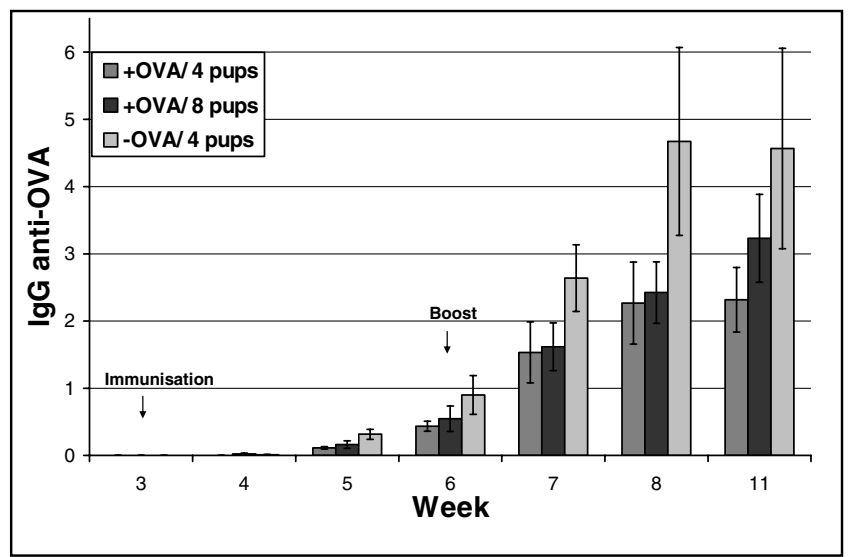

Fig. 1. Anti-OVA IgG titre for the three treatment groups: + OVA/4 pups, + OVA/8 pups and -OVA/4 pups. Antibody levels are expressed in arbitrary ELISA units, calculated from a standard curve obtained with a pool of hyperimmune plasma (mean $\pm \mathrm{SE}$ ). The intra and inter assay CV for the high, medium and low controls were: $3.2-8.2 \%$ and $4.0 \%$, $1.5-3.7 \%$ and $3.3 \%$, and $3-14.1 \%$ and $4.1 \%$, respectively.

At week 11, the -OVA/4 pups group had developed a higher anti-OVA IgG titre $(4.6 \pm 3.94)$ than the +OVA/4 pups and + OVA/8 pups groups $(2.32 \pm 1.36$ and $3.23 \pm 2.62$, respectively), indicating an induction of tolerance in $+\mathrm{OVA} / 4$ pups and $+\mathrm{OVA} / 8$ pups. However, this effect was not statistically significant $(P \geq 0.2951)$, probably due to the low number of dams/pups in each group, and large individual variation. The variation might be due to difficulties in controlling the OVA intake of the dams, and also the weight difference of the pups (gender difference) at immunisation. In conclusion, the oral tolerance model requires some optimisation, i.e., more animals and proper body weight adjustment of the OVA administration, before its application in immunonutrition studies.

1. Lauridsen C, Yong C, Halekoh U et al. (2008) J Sci Food Agric 88, 720-732.

2. Strobel S \& Mowat AM (1998) Immunol Today 19, 173-181. 